\title{
INFINITE DIMENSIONAL QUADRATIC FORMS OVER ALGEBRAIC NUMBER FIELDS
}

\author{
O. T. O'MEARA
}

We shall prove that nondegenerate quadratic forms in a countable number of variables are equivalent over an algebraic number field $F$ if and only if they are equivalent over all real completions of $F$. Thus, in virtue of Savage's description of forms over the real numbers [7], a class of quadratic forms is characterized in our case by determining positive and negative indices at all the real completions of $F$. On the one hand this generalizes to infinite dimensions the MinkowskiHasse-Witt theory of $n$-ary forms, while on the other hand it extends to number fields the countable work of Everett, Ryser and Kaplansky. The results of Everett and Ryser are for positive definite rational forms [5]. Kaplansky [6] deals with arbitrary forms over the rational numbers and also over any field in which every form in $k$ variables represents 1 . As examples of the latter we have the local fields and the function fields in one variable over finite constant fields [1].

We consider a field $F$ (characteristic $\neq 2$ ) acting on a vector space $V$ of at most countable dimensions over $F$. By a quadratic form $q$ on $V$ is meant [4] the mapping obtained by putting $q(x)=b(x, x)$ where $b(x, y)$ is any given bilinear form on $V \times V$. The new bilinear form

$$
s(x, y)=\frac{1}{2}\{b(x, y)+b(y, x)\}
$$

is symmetric and defines the same $q(x)$. Since

$$
s(x, y)=\frac{1}{2}\{q(x+y)-q(x)-q(y)\},
$$

the form $s(x, y)$ is uniquely determined by the given $q$. The forms $q, q^{\prime}$ on $V, V^{\prime}$ are said to be equivalent if there is an isomorphism $\pi$ of $V$ onto $V^{\prime}$ such that $q^{\prime}(\pi x)=q(x)$. This is the same as saying $s(x, y)$ $=s^{\prime}(\pi x, \pi y)$ for all $x$ and $y$ in $V$, by (1). We make it a rule that different forms belong to different spaces so that, without fear of confusing the notation, we can write $x \cdot y=s(x, y)$ and $x^{2}=q(x)$ in $V$. Note that $V \sim V^{\prime}$ if and only if $V$ and $V^{\prime}$ have bases with the same multiplication table. Any symmetric multiplication table defines a unique form on $V$.

All given spaces are assumed nondegenerate. $V=U \oplus W$ means

Received by the editors September 23, 1957 and, in revised form, July 3, 1958. 
that $V$ is the orthogonal direct sum of $U$ and $W$. And $U$ and $W$ are again nondegenerate. If $U$ is any finite dimensional nondegenerate subspace of $V$, then $V=U \oplus U^{\perp}$. It is known that $V$ always has an orthogonal basis but, generally speaking, it need not have an orthonormal one. For further details the reader is referred to Kaplansky [6].

Let $F_{\mathfrak{a}}$ be an extension of $F$. We call the metric $F_{\mathfrak{a}}$-space $V_{\mathfrak{a}}$ an extension of $V$ with respect to $F_{\mathfrak{a}}$ if $V_{\mathbf{a}}$ is generated by $V$. Since $V$ is nondegenerate, any orthogonal basis for $V$ is an orthogonal basis for $V_{\mathfrak{a}}$ so that any two extensions of $V$ are isometric. That $V$ always has an extension is easily seen by constructing the $F_{a}$-space with basis $\langle x\rangle$ and metric given by $\left(x_{i} \cdot x_{j}\right)$. If $U$ and $V$ are isometric, then so are $U_{\mathfrak{a}}$ and $V_{\mathfrak{a}}$.

From now on $F$ will be a finite algebraic number field. We denote by $Q$ the total set of inequivalent primes of $F$ and we let $S$ be the finite set of real primes in $Q$. We use ||$_{p}$ for a valuation and $F_{p}$ for the completion associated with the prime $\mathfrak{p}$. It follows from [6] that $V_{p}$ has an orthonormal basis for all finite or complex primes. For a real $\mathfrak{p}$ there is a decomposition $V_{\mathfrak{p}}=U \oplus V$ in which $U$ and $(-1 \circ W)$ have orthonormal bases, and we call $p_{\mathfrak{p}}(V)=\operatorname{dim} U, n_{\mathfrak{p}}(V)=\operatorname{dim} V$ the positive and negative indices at $\mathfrak{p}$. Note that $V_{\mathfrak{p}} \sim V_{\mathfrak{p}}^{\prime}$ if and only if their indices are the same at $\mathfrak{p}$, by [7]. For any orthogonal basis $\langle x\rangle$ of $V$, the positive index at $\mathfrak{p}$ is the number of positive $x^{2} \in F_{\mathfrak{p}}$ obtained by letting $x$ run through $\langle x\rangle$; and similarly with the negative index.

THEOREM. The countable dimensional spaces $V$ and $V^{\prime}$ are isometric if and only if they have the same positive and negative indices at all real primes of $F$.

Proof. We need only prove the sufficiency. Write $V$ in the orthogonal basis $V=\oplus_{1}^{\infty} F x_{\mu}$. We shall first show how to reduce the problem to the case where

$$
p_{\mathfrak{p}}(V)=0 \text { or } \infty, \quad n_{\mathfrak{p}}(V)=0 \text { or } \infty
$$

holds at all $p \in S$. To this end let us suppose that $0<p_{\mathfrak{q}}(V)<\infty$ holds for some particular prime $q \in S$. Using the Approximation Theorem [2] we find an $\epsilon \in F$ such that

$$
\begin{array}{ll}
|\epsilon-1|_{\mathfrak{p}}<1 & \text { when } p_{\mathfrak{p}}(V)>0, \mathfrak{p} \in S, \\
|\epsilon+1|_{\mathfrak{p}}<1 & \text { at all other } \mathfrak{p} \in S .
\end{array}
$$

Thus $\epsilon \in F_{\mathfrak{p}}$ is positive in the first instance, negative in the second. Choose $j \geqq 5$ and so large that $p_{\mathfrak{p}}(U)>0$ whenever $p_{\mathfrak{p}}(V)>0$ with 
$\mathfrak{p} \in S$, where $U=F x_{1} \oplus \cdots \oplus F x_{j}$. Then $\epsilon \in U_{\mathfrak{p}}^{2}$ at all $\mathfrak{p} \in S$ in virtue of the definitions of $\epsilon$ and $U$, and $\epsilon \in U_{p}^{2}$ when $p \in S$ by Theorems 15 and 16 of [8]. Hence $\epsilon=x^{2} \in U^{2} \subseteq V^{2}$ by Theorems 15 and 19 of [8]. Since $F x$ is finite dimensional we have

$$
V=(\epsilon) \oplus \bar{V}, \quad V^{\prime}=(\epsilon) \oplus \bar{V}^{\prime},
$$

the second equation following by symmetry. Then $p_{\mathfrak{p}}(\bar{V})=p_{\mathfrak{p}}\left(\bar{V}^{\prime}\right)$ and $n_{\mathfrak{p}}(\bar{V})=n_{\mathfrak{p}}\left(\bar{V}^{\prime}\right)$ hold at all $\mathfrak{p} \in S$, while

$$
p_{\mathrm{a}}\left(\bar{V}^{\prime}\right)=p_{\mathrm{a}}(\bar{V})<p_{\mathrm{a}}(V)=p_{\mathrm{a}}\left(V^{\prime}\right) .
$$

Repeat this until the positive index at $q$ is 0 . Now do the same thing at all other primes with finite positive or negative index. After a finite number of steps we get $V=W_{1} \oplus W, V^{\prime}=W_{1}^{\prime} \oplus W^{\prime}$ with $W_{1} \sim W_{1}^{\prime}$ and

$$
p_{\mathfrak{p}}(W)=p_{\mathfrak{p}}\left(W^{\prime}\right)=0 \text { or } \infty, \quad n_{\mathfrak{p}}(W)=n_{\mathfrak{p}}\left(W^{\prime}\right)=0 \text { or } \infty
$$

at all $\mathfrak{p} \in S$. In effect, this allows us to assume that $V$ and $V^{\prime}$ satisfy (2). We make this assumption from now on. This ends the reduction of the problem.

Let $T$ denote those real primes at which $V$ and $V^{\prime}$ have both indices infinite, let $P$ denote the primes for which $n_{\mathfrak{p}}(V)=0=n_{\mathfrak{p}}\left(V^{\prime}\right)$, let $N$ denote the primes for which $p_{\mathfrak{p}}(V)=0=p_{\mathfrak{p}}\left(V^{\prime}\right)$. Thus $S$ is the disjoint union $S=T \cup P \cup N$. Now use the Approximation Theorem to define an $\epsilon_{1} \in F$ such that

$$
\left|\epsilon_{1}-e_{\mathfrak{p}}\right|_{\mathfrak{p}}<1 \text { where } e_{\mathfrak{p}}=\left\{\begin{array}{l}
+1 \text { if } \mathfrak{p} \in T \cup P, \\
-1 \text { if } \mathfrak{p} \in N
\end{array}\right.
$$

Similarly define an $\epsilon_{2} \in F$ such that

$$
\left|\epsilon_{2}-e_{\mathfrak{p}}\right|_{\mathfrak{p}}<1 \text { where } e_{\mathfrak{p}}=\left\{\begin{array}{l}
-1 \text { if } \mathfrak{p} \in T \cup N, \\
+1 \text { if } \mathfrak{p} \in P .
\end{array}\right.
$$

We put $\epsilon_{2}=\epsilon_{3}=\epsilon_{4}=\epsilon_{5}$. We shall show that $V$ has a basis

$$
V=\oplus_{1}^{\infty} F X_{\mu} \quad \text { with } \quad X_{\mu}^{2}=\epsilon_{i} \quad \text { for } \quad \mu \equiv i \bmod 5 .
$$

Suppose that $X_{1}, \cdots, X_{5 k}$ have been defined for $k \geqq 0$ in such a way that

$$
\begin{array}{rlrl}
x_{j} \in U & =\bigoplus_{1}^{5 k} F X_{\mu} & \text { for } 1 \leqq j \leqq k, \\
X_{\mu}^{2}=\epsilon_{i} & \text { for } \mu \equiv i \bmod 5 .
\end{array}
$$


We must define $X_{5 k+1}, \cdots, X_{5(k+1)}$. Let $x_{8}$ be the first element of the basis $\langle x\rangle$ which is not in $U$. So $s \geqq k+1$. Since $U$ is finite dimensional we can write $V=U \oplus U^{\perp}$. Write $x_{s}=y+z$ with $y \in U, z \in U^{\perp}$, and then put $H=F z$ if $z^{2} \neq 0$, and put $H=F z+F z^{\prime}$ with $z^{\prime} \in U^{\perp}, z \cdot z^{\prime} \neq 0$, if $z^{2}=0$. In the latter case $H$ is a hyperbolic plane. We write $V=U \oplus H$ $\oplus U^{\prime}$. Now it is easily verified, using Theorems 15,16 and 19 of [8], that the quadratic form $\epsilon_{1} \oplus \cdots \oplus \epsilon_{5}$ represents $H$. Hence

$$
\epsilon_{1} \oplus \cdots \oplus \epsilon_{5} \sim H \oplus J .
$$

On the other hand, any space with the same indices as $V$ must represent $\epsilon_{1}, \epsilon_{2}$ (indeed, any sufficiently large finite dimensional subspace will do so) and hence it must represent $\epsilon_{1} \oplus \cdots \oplus \epsilon_{5}$; in particular, $U^{\prime}$ has a component $J$. Therefore $V=U \oplus(H \oplus J) \oplus U^{\prime \prime}$. Using (5) we define $X_{5 k+1}, \cdots, X_{5(k+1)}$ so that $H \oplus J=\oplus_{1}^{5} F X_{5 k+\mu}$ and $X_{2 k+\mu}^{5}=\epsilon_{\mu}$. This has the required properties. In this way define $X_{\mu}(1 \leqq \mu<\infty)$. This is a basis for $V$ which satisfies (3).

Obviously all this can be done for $V^{\prime}$ too. Thus

$$
V^{\prime}=\oplus_{1}^{\infty} F X_{\mu}^{\prime} \quad \text { with } \quad\left(X_{\mu}^{\prime}\right)^{2}=\epsilon_{i} \quad \text { for } \mu \equiv i \bmod 5 .
$$

Hence $V \sim V^{\prime}$. This proves the theorem.

\section{REFERENCES}

1. A. A. Albert, Quadratic null forms over a function field, Ann. of Math. (2) vol. 39 (1938) pp. 494-505.

2. E. Artin, Algebraic numbers and algebraic functions, Princeton, 1950.

3. N. Bourbaki, Algèbre linéaire, Eléments de Mathématique, Paris, 1947.

4. C. Chevalley, The algebraic theory of spinors, New York, 1954.

5. C. J. Everett and H. J. Ryser, Rational vector spaces I, Duke Math. J. vol. 16 (1949) pp. 553-570.

6. I. Kaplansky, Forms in infinite-dimensional spaces, An. Acad. Brasil. Ci. vol. 22 (1950) pp. 1-17.

7. L. J. Savage, The application of vectorial methods to metric geometry, Duke Math. J. vol. 13 (1946) pp. 521-528.

8. E. Witt, Theorie der quadratischen Formen in beliebigen Körpern, J. Reine Angew. Math. vol. 176 (1937) pp. 31-44.

Princeton University 